PROCEEDINGS OF THE

AMERICAN MATHEMATICAL SOCIETY

Volume 127, Number 8, Pages 2283-2288

S 0002-9939(99)04819-4

Article electronically published on April 9, 1999

\title{
A STRUCTURE OF RING HOMOMORPHISMS ON COMMUTATIVE BANACH ALGEBRAS
}

\author{
SIN-EI TAKAHASI AND OSAMU HATORI \\ (Communicated by David R. Larson)
}

Dedicated to Professor Jyunji Inoue on his sixtieth birthday

\begin{abstract}
We give a structure theorem for a ring homomorphism of a commutative regular Banach algebra into another commutative Banach algebra. In particular, it is shown that:

(i) A ring homomorphism of a commutative $\mathrm{C}^{*}$-algebra onto another commutative $\mathrm{C}^{*}$-algebra with connected infinite Gelfand space is either linear or anti-linear.

(ii) A ring automorphism of $L^{1}\left(\boldsymbol{R}^{N}\right)$ is either linear or anti-linear.

(iii) $C^{n}([a, b]), L^{1}\left(\boldsymbol{R}^{N}\right)$ and the disc algebra $A(D)$ are neither ring homomorphic images of $\ell^{1}(S)$ nor $L^{p}(G)(1 \leq p<\infty, G$ a compact abelian group).
\end{abstract}

Let $A$ and $B$ be two commutative Banach algebras with Gelfand spaces $\Phi_{A}$ and $\Phi_{B}$, respectively. Let $\rho$ be a ring homomorphism of $A$ into $B$ such that

$(*)$

$$
\left\{\rho(x)^{\wedge}(\psi): x \in A\right\}=\boldsymbol{C}, \quad \text { the complex field, }
$$

for each $\psi \in \Phi_{B}$ ("^" denotes the Gelfand transform). This, of course, holds if $\rho$ is onto.

The purpose of this note is to show the following structure theorem of $\rho$ applying the method which L. Molnar used in [5] to prove that a commutative semisimple Banach algebra which is the range of a ring homomorphism from a commutative $\mathrm{C}^{*}$-algebra must be $\mathrm{C}^{*}$-equivalent.

Theorem 1. Suppose $A$ is regular. Then there exist a continuous map $\hat{\rho}$ of $\Phi_{B}$ into $\Phi_{A}$ and a division $\left\{\Phi_{B}^{0}, \Phi_{B}^{1}, \Phi_{B}^{2}\right\}$ of $\Phi_{B}$ such that $\Phi_{B}^{1}$ and $\Phi_{B}^{2}$ are closed, and for each $a \in A, \rho(a)^{\wedge}=\hat{a} \circ \hat{\rho}$ on $\Phi_{B}^{1}, \rho(a)^{\wedge}=\overline{\hat{a}} \circ \hat{\rho}$ on $\Phi_{B}^{2}$ and $\rho(a)^{\wedge}(\psi)=\tau_{\psi}(\hat{a}(\hat{\rho}(\psi)))$ for every $\psi \in \Phi_{B}^{0}$ and for a certain discontinuous ring automorphism $\tau_{\psi}$ of the complex field $\boldsymbol{C}$.

Moreover, if $\rho$ is surjective, then $\hat{\rho}$ is injective, and if $A$ satisfies the following condition $(\#)$, then $\hat{\rho}\left(\Phi_{B}^{0}\right)$ is a finite set:

(\#) For any $\lambda_{n} \in \boldsymbol{C}$ with $\left|\lambda_{n}\right| \leq 1 / 2^{n}(n=1,2, \ldots)$ and any sequence $\left\{\varphi_{1}, \varphi_{2}, \ldots\right\}$ in $\Phi_{A}$ such that each $\varphi_{n}$ is an isolated point in $\left\{\varphi_{1}, \varphi_{2}, \ldots\right\}$, there exists an element $a \in A$ such that $\hat{a}\left(\varphi_{n}\right)=\lambda_{n}(n=1,2, \ldots)$.

Received by the editors May 29, 1997 and, in revised form, October 27, 1997.

1991 Mathematics Subject Classification. Primary 46J05, 46E25.

Key words and phrases. Commutative Banach algebra, ring homomorphism, Gelfand transform, Fourier transform.

The authors are partly supported by the Grants-in-Aid for Scientific Research, The Ministry of Education, Science and Culture, Japan. 
Remark 1. If $A$ is a commutative regular Banach algebra which satisfies the condition (\#) and if $\rho$ is surjective, then $\Phi_{B}^{0}$ is a finite set and hence both $\Phi_{B}^{1}$ and $\Phi_{B}^{2}$ are clopen.

Remark 2. A commutative C $\mathrm{C}^{*}$-algebra, $\ell^{1}(S)(S$ a set $), L^{1}\left(\boldsymbol{R}^{N}\right)$ and $L^{p}(G)(1 \leq$ $p<\infty, G$ a compact abelian group) are commutative regular Banach algebras which satisfy the condition (\#). The details of these algebras can be seen just before Corollary 4.

Now in order to prove the theorem, we have to prepare some lemmas.

Lemma 1. If $I$ is a closed ideal of $B$ such that $I=\bigcap_{\substack{\subseteq \subseteq \operatorname{Ker}(\psi) \\ \psi \in \Phi_{B}}} \operatorname{Ker}(\psi)$, then $\rho^{-1}(I)$ is a closed algebra ideal of $A$.

Proof. We first show that $\rho^{-1}(I)$ is norm-closed. To do this, let $a$ be any element in the norm-closure of $\rho^{-1}(I)$. We will show that

$$
\rho(a)^{\wedge}(\psi) \rho(x)^{\wedge}(\psi) \neq 1
$$

for all $x \in A$ and $\psi \in \Phi_{B}$ with $I \subseteq \operatorname{Ker}(\psi)$. Actually, for any arbitrary element $x \in A$, choose an element of $y \in \rho^{-1}(I)$ such that $\|a x-y\|<1$ since $a x$ belongs to the norm-closure of $\rho^{-1}(I)$, and define

$$
z=\sum_{n=1}^{\infty}(a x-y)^{n} .
$$

We have $z a x-z y=z-(a x-y)$ and hence $(\rho(z)+1) \rho(a) \rho(x)-\rho(z) \in I$. This implies easily that $\rho(a)^{\wedge}(\psi) \rho(x)^{\wedge}(\psi) \neq 1$ for all $\psi \in \Phi_{B}$ with $I \subseteq \operatorname{Ker}(\psi)$. Let us show now that $a \in \rho^{-1}(I)$. Suppose, on the contrary, that $\rho(a) \notin I$. Since $I=\bigcap_{I \subseteq \operatorname{Ker}(\psi)} \operatorname{Ker}(\psi)$, there exists an element $\psi_{0} \in \Phi_{B}$ such that $I \subseteq \operatorname{Ker}\left(\psi_{0}\right)$ and $\rho(a)^{\wedge}\left(\psi_{0}\right) \neq 0$. Then we can choose an element $x_{0} \in A$ such that $\rho\left(x_{0}\right)^{\wedge}\left(\psi_{0}\right)=$ $\frac{1}{\rho(a)^{\wedge}\left(\psi_{0}\right)}$ since $\rho$ satisfies the condition $(*)$. But this contradicts $(1)$ and then $\rho^{-1}(I)$ is norm-closed.

We next show that $\rho^{-1}(I)$ is an algebra ideal of $A$. To do this, let $x \in \rho^{-1}(I)$ and $\lambda \in \boldsymbol{C}$. Choose a sequence $\left\{\alpha_{1}, \beta_{1}, \alpha_{2}, \beta_{2}, \ldots\right\}$ of rational numbers such that $\lim _{n \rightarrow \infty}\left(\alpha_{n}+i \beta_{n}\right)=\lambda$. Then we have $\lim _{n \rightarrow \infty}\left\|\alpha_{n} x+i \beta_{n} x-\lambda x\right\|=0$ and $\alpha_{n} x, \beta_{n} x \in \rho^{-1}(I)$ for each integer $n \geq 1$. For any $\psi \in \Phi_{B}$ with $I \subseteq \operatorname{Ker}(\psi)$, we have

$$
\psi\left(\rho\left(i \beta_{n} x\right)\right)^{2}=-\psi\left(\rho\left(\beta_{n} x\right)\right)^{2}=0,
$$

and hence $\psi\left(\rho\left(i \beta_{n} x\right)\right)=0$. Then $\rho\left(i \beta_{n} x\right) \in I$ since $I=\bigcap_{I \subseteq \operatorname{Ker}(\psi)} \operatorname{Ker}(\psi)$. Therefore we have $\alpha_{n} x+i \beta_{n} x \in \rho^{-1}(I)$ for each integer $n \geq 1$, and hence $\lambda x \in \rho^{-1}(I)$ since $\rho^{-1}(I)$ is norm-closed.

Q.E.D.

Lemma 2. There exists a mapping $\hat{\rho}$ of $\Phi_{B}$ into $\Phi_{A}$ such that

$$
\rho(a)^{\wedge}(\psi)=\tau_{\psi}(\hat{a}(\hat{\rho}(\psi))) \quad(a \in A)
$$

for every $\psi \in \Phi_{B}$ and for a certain ring automorphism $\tau_{\psi}$ of $\boldsymbol{C}$.

Proof. Let $\psi$ be any element of $\Phi_{B}$ and define $\rho_{\psi}(a)=\rho(a)^{\wedge}(\psi)$ for each $a \in A$. Then $\rho_{\psi}$ is a ring homomorphism of $A$ onto $C$ by the condition (*). Hence we can easily see that $\operatorname{Ker}\left(\rho_{\psi}\right)$ is a closed algebra ideal of $A$ by applying the preceding lemma. From this, $A / \operatorname{Ker}\left(\rho_{\psi}\right)$ is a unital commutative Banach algebra which is ring isomorphic to $\boldsymbol{C}$. Then there exists an algebra homomorphism of $A / \operatorname{Ker}\left(\rho_{\psi}\right)$ 
onto $\boldsymbol{C}$, say $\eta$. Let $\varphi$ be the composition map of the canonical map from $A$ onto $A / \operatorname{Ker}\left(\rho_{\psi}\right)$ and $\eta$. Then $\varphi$ is an element of $\Phi_{A}$ such that $\operatorname{Ker}\left(\rho_{\psi}\right) \subseteq \operatorname{Ker}(\varphi)$. Since $\boldsymbol{C}$ is a simple ring and $A / \operatorname{Ker}\left(\rho_{\psi}\right)$ is ring isomorphic to $\boldsymbol{C}$, it follows that $\operatorname{Ker}\left(\rho_{\psi}\right)=\operatorname{Ker}(\varphi)$. Set $\hat{\rho}(\psi)=\varphi$. Then $\hat{\rho}$ is a mapping of $\Phi_{B}$ into $\Phi_{A}$ and we have

$$
\begin{aligned}
\boldsymbol{C} \cong A / \operatorname{Ker}(\hat{\rho}(\psi)) & =A / \operatorname{Ker}\left(\rho_{\psi}\right) \cong \boldsymbol{C}, \\
\hat{a}(\hat{\rho}(\psi)) \leftrightarrow a+\operatorname{Ker}(\hat{\rho}(\psi)) & =\dot{a}+\operatorname{Ker}\left(\rho_{\psi}\right) \leftrightarrow \rho(a)^{\wedge}(\psi)
\end{aligned}
$$

for each $a \in A$, where the former is an algebra isomorphism and the latter is a ring isomorphism. Therefore a desired map $\tau_{\psi}$ can be obtained as the above composition map from $\boldsymbol{C}$ onto itself.

Q.E.D.

Lemma 3. If $A$ is regular, then $\hat{\rho}$ is continuous on $\Phi_{B}$.

Proof. Let $\psi$ be any element of $\Phi_{B},\left\{\psi_{\lambda}\right\}$ any net in $\Phi_{B}$ which converges to $\psi$ and $U$ any open neighbourhood of $\hat{\rho}(\psi)$. Suppose that $A$ is regular. Then we can find an element $a$ of $A$ such that $\hat{a}(\hat{\rho}(\psi))=1$ and $\hat{a}=0$ on $\Phi_{A} \backslash U$. By the preceding lemma, we have

$$
\lim _{\lambda} \tau_{\psi_{\lambda}}\left(\hat{a}\left(\hat{\rho}\left(\psi_{\lambda}\right)\right)\right)=\lim _{\lambda} \rho(a)^{\wedge}\left(\psi_{\lambda}\right)=\rho(a)^{\curlywedge}(\psi)=\tau_{\psi}(\hat{a}(\hat{\rho}(\psi)))=\tau_{\psi}(1)=1 .
$$

Then there exists a $\lambda_{0}$ such that

$$
\tau_{\psi_{\lambda}}\left(\hat{a}\left(\hat{\rho}\left(\psi_{\lambda}\right)\right)\right) \neq 0, \quad \text { i.e., } \hat{a}\left(\hat{\rho}\left(\psi_{\lambda}\right)\right) \neq 0 \text { and so } \hat{\rho}\left(\psi_{\lambda}\right) \in U
$$

for every $\lambda \geq \lambda_{0}$. This means $\lim _{\lambda} \hat{\rho}\left(\psi_{\lambda}\right)=\hat{\rho}(\psi)$ and the proof is complete. Q.E.D.

Lemma 4. If $\rho$ is surjective, then $\hat{\rho}$ is injective and condition $(*)$ holds automatically.

Proof. Suppose that there exist two elements $\psi_{1}$ and $\psi_{2}$ in $\Phi_{B}$ such that $\psi_{1} \neq \psi_{2}$ and $\hat{\rho}\left(\psi_{1}\right)=\hat{\rho}\left(\psi_{2}\right)\left(\equiv \varphi \in \Phi_{A}\right)$. By Lemma 2, we have

$$
\rho(a)^{\wedge}\left(\psi_{1}\right)=\tau_{\psi_{1}}\left(\hat{a}\left(\hat{\rho}\left(\psi_{1}\right)\right)\right)=\tau_{\psi_{1}}(\varphi(a))=\tau_{\psi_{1}}(0)=0
$$

for every $a \in \operatorname{Ker}(\varphi)$, and hence $\rho(\operatorname{Ker}(\varphi)) \subseteq \operatorname{Ker}\left(\psi_{1}\right)$. Similarly we have $\rho(\operatorname{Ker}(\varphi))$ $\subseteq \operatorname{Ker}\left(\psi_{2}\right)$ and so $\rho(\operatorname{Ker}(\varphi)) \subseteq \operatorname{Ker}\left(\psi_{1}\right) \cap \operatorname{Ker}\left(\psi_{2}\right)$. But since $\psi_{1} \neq \psi_{2}$, it follows that $\operatorname{Ker}\left(\psi_{1}\right) \cap \operatorname{Ker}\left(\psi_{2}\right) \varsubsetneqq \operatorname{Ker}\left(\psi_{1}\right)$. Then we obtain

$$
\rho(\operatorname{Ker}(\varphi)) \varsubsetneqq \operatorname{Ker}\left(\psi_{1}\right) \text {. }
$$

Also we have

$$
\operatorname{Ker}(\rho) \subseteq \operatorname{Ker}(\varphi)
$$

since $\varphi(a)=\hat{a}\left(\hat{\rho}\left(\psi_{1}\right)\right)=\tau_{\psi_{1}}^{-1}\left(\rho(a)^{\wedge}\left(\psi_{1}\right)\right)=\tau_{\psi_{1}}^{-1}(0)=0$ for every $a \in \operatorname{Ker}(\rho)$. Therefore if $\rho$ is surjective, then we have

$$
\begin{aligned}
C & \cong A / \operatorname{Ker}(\varphi) \quad(\text { algebra isomorphic) } \\
& \cong(A / \operatorname{Ker}(\rho)) /(\operatorname{Ker}(\varphi) / \operatorname{Ker}(\rho)) \quad(\text { ring isomorphic) (by }(3)) \\
& \cong B / \rho(\operatorname{Ker}(\varphi)) \quad(\text { ring isomorphic) } \\
& \supsetneqq \operatorname{Ker}\left(\psi_{1}\right) / \rho(\operatorname{Ker}(\varphi)) \\
& \supsetneqq\{0\} \quad(\text { by }(2)) .
\end{aligned}
$$

But this is a contradiction since $\boldsymbol{C}$ is a simple ring and the proof is complete.

Q.E.D. 
We are now in a position to prove our main theorem.

Proof of Theorem 1. Let us consider the following three sets:

$$
\begin{aligned}
& \Phi_{B}^{0}=\left\{\psi \in \Phi_{B}: \tau_{\psi} \text { is discontinuous }\right\}, \\
& \Phi_{B}^{1}=\left\{\psi \in \Phi_{B}: \tau_{\psi}(\lambda)=\lambda \text { for all } \lambda \in C\right\}, \\
& \Phi_{B}^{2}=\left\{\psi \in \Phi_{B}: \tau_{\psi}(\lambda)=\bar{\lambda} \text { for all } \lambda \in C\right\} .
\end{aligned}
$$

In this case, it is easy to see that

$$
\Phi_{B}=\Phi_{B}^{0} \cup \Phi_{B}^{1} \cup \Phi_{B}^{2} \quad \text { (disjoint union). }
$$

Moreover, by definition and Lemma 2, we have that for each $a \in A, \rho(a)^{\wedge}=\hat{a} \circ \hat{\rho}$ on $\Phi_{B}^{1}, \rho(a)^{\wedge}=\overline{\hat{a}} \circ \hat{\rho}$ on $\Phi_{B}^{2}$ and $\rho(a)^{\wedge}(\psi)=\tau_{\psi}(\hat{a}(\hat{\rho}(\psi)))$ for every $\psi \in \Phi_{B}^{0}$ and for a certain discontinuous ring automorphism $\tau_{\psi}$ of $\boldsymbol{C}$.

Assume now that $A$ is regular. Then $\hat{\rho}$ is continuous on $\Phi_{B}$ by Lemma 3 . We shall show that $\Phi_{B}^{1}$ is closed in $\Phi_{B}$. To do this, let $\left\{\psi_{\lambda}\right\}$ be a net in $\Phi_{B}^{1}$ which converges to an element $\psi \in \Phi_{B}$. Then

$$
\hat{a}(\hat{\rho}(\psi))=\lim _{\lambda} \hat{a}\left(\hat{\rho}\left(\psi_{\lambda}\right)\right)=\lim _{\lambda} \rho(a)^{\wedge}\left(\psi_{\lambda}\right)=\rho(a)^{\wedge}(\psi)
$$

for all $a \in A$. Therefore we have

$$
\tau_{\psi}\left(\rho(a)^{\curlywedge}(\psi)\right)=\tau_{\psi}(\hat{a}(\hat{\rho}(\psi)))=\rho(a)^{\curlywedge}(\psi)
$$

for all $a \in A$. By the above fact and the condition $(*)$, we have $\tau_{\psi}(\lambda)=\lambda$ for every $\lambda \in C$. In other words, $\psi \in \Phi_{B}^{1}$ and hence $\Phi_{B}^{1}$ is closed in $\Phi_{B}$. Similarly, it is shown that $\Phi_{B}^{2}$ is also closed in $\Phi_{B}$.

Let us assume that $A$ additionally satisfies the condition (\#). We shall show that $\hat{\rho}\left(\Phi_{B}^{0}\right)$ is a finite set. Suppose not. Then we can choose a sequence $\left\{\varphi_{1}, \varphi_{2}, \ldots\right\}$ in $\hat{\rho}\left(\Phi_{B}^{0}\right)$ such that each $\varphi_{n}$ is an isolated point of the subset $\left\{\varphi_{1}, \varphi_{2}, \ldots\right\}$ (not necessarily an isolated point of $\hat{\rho}\left(\Phi_{B}^{0}\right)$ ). For each $\varphi_{n}$, choose an element $\psi_{n}$ of $\Phi_{B}^{0}$ with $\varphi_{n}=\hat{\rho}\left(\psi_{n}\right)$. Since each $\tau_{\psi_{n}}$ is a discontinuous automorphism of $C$, it follows from $\left[2\right.$, Theorem 2, p. 360] that $\tau_{\psi_{n}}$ maps every disc onto an unbounded set and hence we can take a complex number $\lambda_{n}$ such that $\left|\lambda_{n}\right| \leq \frac{1}{2^{n}}$ and $\left|\tau_{\psi_{n}}\left(\lambda_{n}\right)\right| \geq n$. By the condition (\#), there exists an element $a \in A$ such that $\hat{a}\left(\varphi_{n}\right)=\lambda_{n}$ for each integer $n \geq 1$.

Therefore we have

$$
\left|\rho(a)^{\wedge}\left(\psi_{n}\right)\right|=\left|\tau_{\psi_{n}}\left(\hat{a}\left(\hat{\rho}\left(\psi_{n}\right)\right)\right)\right|=\left|\tau_{\psi_{n}}\left(\hat{a}\left(\varphi_{n}\right)\right)\right|=\left|\tau_{\psi_{n}}\left(\lambda_{n}\right)\right| \geq n
$$

for each integer $n \geq 1$. On the other hand, we have

$$
\left|\rho(a)^{\wedge}\left(\psi_{n}\right)\right| \leq\|\rho(a)\|
$$

for each integer $n \geq 1$. This is a contradiction.

Q.E.D.

Corollary 1. Every ring homomorphism of a commutative $\mathrm{C}^{*}$-algebra onto another commutative $\mathrm{C}^{*}$-algebra with connected infinite Gelfand space is either linear or anti-linear.

Proof. Since every commutative $\mathrm{C}^{*}$-algebra is a regular Banach algebra which satisfies the condition (\#), the corollary follows immediately from Theorem 1. Q.E.D.

Corollary 2. Every ring homomorphism of a commutative $\mathrm{C}^{*}$-algebra onto another commutative $\mathrm{C}^{*}$-algebra whose Gelfand space has no isolated points is continuous. 
Proof. Let $A$ and $B$ be two commutative $\mathrm{C}^{*}$-algebras and $\rho$ a ring homomorphism of $A$ onto $B$. Assume that the Gelfand space $\Phi_{B}$ of $B$ has no isolated points. By Theorem 1, we can find two clopen subsets $\Phi_{B}^{1}$ and $\Phi_{B}^{2}$ of $\Phi_{B}$ such that $\Phi_{B}^{1} \cap \Phi_{B}^{2}=$ $\varnothing, \Phi_{B}^{1} \cup \Phi_{B}^{2}=\Phi_{B}$ and $\rho(a)^{\wedge}=\hat{a} \circ \hat{\rho}$ on $\Phi_{B}^{1}, \rho(a)^{\wedge}=\overline{\hat{a}} \circ \hat{\rho}$ on $\Phi_{B}^{2}$ for each $a \in A$. Set

$$
I_{1}=\left\{x \in B: \hat{x}=0 \text { on } \Phi_{B}^{1}\right\} \quad \text { and } \quad I_{2}=\left\{x \in B: \hat{x}=0 \text { on } \Phi_{B}^{2}\right\} .
$$

Then $I_{1}$ and $I_{2}$ are closed ideals of $B$ such that $\Phi_{B / I_{1}} \cong \Phi_{B}^{1}$ and $\Phi_{B / I_{2}} \cong \Phi_{B}^{2}$. Let $\rho_{1}$ (resp. $\rho_{2}$ ) be the composition map of the canonical map of $B$ onto $B / I_{1}$ (resp. $\left.B / I_{2}\right)$ and $\rho$. Then it is easy to see that $\rho_{1}$ is an algebra homomorphism of $A$ onto $B / I_{1}$ and $\rho_{2}$ is an anti-algebra homomorphism of $A$ onto $B / I_{2}$. Hence both $\rho_{1}$ and $\rho_{2}$ are continuous by the Johnson theorem [1]. On the other hand, observe that

$$
\|\rho(a)\|=\max \left(\left\|\rho_{1}(a)\right\|,\left\|\rho_{2}(a)\right\|\right)
$$

for all $a \in A$. Therefore $\rho$ must be continuous.

Q.E.D.

Lemma 5. The group algebra $L^{1}\left(\boldsymbol{R}^{N}\right)$ satisfies the condition (\#).

Proof. Choose a function $f \in L^{1}\left(\boldsymbol{R}^{N}\right)$ such that $\hat{f}(0)=1$ and $\hat{f}(\xi)=0$ for each $\xi \in \boldsymbol{R}^{N}$ with $\|\xi\| \geq 1$, where $\hat{f}$ denotes the Fourier transform of $f$. For any fixed $\alpha>0$ and $b \in \boldsymbol{R}^{N}$, set

$$
f_{a, b}(x)=\alpha^{-N} f\left(\alpha^{-1} x\right) e^{-i \alpha^{-1}\langle b, x\rangle}
$$

for each $x \in \boldsymbol{R}^{N}$, where $\langle b, x\rangle$ denotes the inner product of $b$ and $x$. Then a simple calculation implies that $\left\|f_{a, b}\right\|_{1}=\|f\|_{1}$ and $\hat{f}_{a, b}(\xi)=\hat{f}(\alpha \xi+b)$ for every $\xi \in \boldsymbol{R}^{N}$.

Now to show that $L^{1}\left(\boldsymbol{R}^{N}\right)$ satisfies the condition (\#), let $\lambda_{n} \in \boldsymbol{C}$ with $\left|\lambda_{n}\right| \leq$ $1 / 2^{n}(n=1,2, \ldots)$ and $\left\{\xi_{1}, \xi_{2}, \ldots\right\} \subseteq \boldsymbol{R}^{N}$ such that each $\xi_{n}$ is an isolated point in $\left\{\xi_{1}, \xi_{2}, \ldots\right\}$. Set

$$
\alpha_{n}=\sup _{n \neq k}\left\|\xi_{n}-\xi_{k}\right\|^{-1} \quad(n=1,2, \ldots) .
$$

Then all $\alpha_{n}$ are positive numbers since each $\xi_{n}$ is an isolated point in $\left\{\xi_{1}, \xi_{2}, \ldots\right\}$. Consider the following function $g(x)$ on $\boldsymbol{R}^{N}$ defined by

$$
g(x)=\sum_{n=1}^{\infty} \lambda_{n} f_{\alpha_{n},-\alpha_{n} \xi_{n}}(x) \quad\left(x \in \boldsymbol{R}^{N}\right) .
$$

Since $\left\|\lambda_{n} f_{\alpha_{n},-\alpha_{n} \xi_{n}}\right\|_{1}=\left|\lambda_{n}\right|\|f\|_{1} \leq \frac{1}{2^{n}}\|f\|_{1}(n=1,2, \ldots)$, it follows that $g$ belongs to $L^{1}\left(\boldsymbol{R}^{N}\right)$ and $\hat{g}\left(\xi_{n}\right)=\lambda_{n}(n=1,2, \ldots)$ by the simple calculation. In other words, $L^{1}\left(\boldsymbol{R}^{N}\right)$ satisfies the condition (\#).

Q.E.D.

Corollary 3. A ring automorphism of $L^{1}\left(\boldsymbol{R}^{N}\right)$ is either linear or anti-linear.

Proof. This is an immediate consequence of Theorem 1 and Lemma 5. Q.E.D.

For each nonnegative integer $n$, let $C^{n}([a, b])$ denote the family of all $n$-times continuously differentiable complex-valued functions defined on the closed interval $[a, b]$ on $\boldsymbol{R}$. Then $C^{n}([a, b])$ becomes a semisimple commutative Banach algebra in the usual way and its Gelfand space is homeomorphic to $[a, b]$ (cf. Larsen [4, p. 92]). Let $G$ be a compact abelian group and $1 \leq p<\infty$. Then the $L^{p}$-space $L^{p}(G)$ on $G$ becomes a semisimple commutative Banach algebra under convolution and its Gelfand space is homeomorphic to the dual group of $G$ (cf. Larsen [3, p. 250]). Let $S$ be a set and $\ell^{1}(S)$ the family of all complex-valued functions $f$ on $S$ such that 
$\|f\|_{1}=\sum_{s \in S}|f(s)|<\infty$. Then $\ell^{1}(S)$ becomes a semisimple commutative Banach algebra under the pointwise operations and the norm $\|f\|_{1}$ and its Gelfand space is homeomorphic to $S$ endowed with the discrete topology (cf. [6, p. 611]).

For these algebras, we have the following result which is similar to Molnar's result [5, Corollary] which asserts that the group algebras $L^{1}(\boldsymbol{R}), L^{1}(\boldsymbol{T})$ and the disc algebra $A(\boldsymbol{D})$ are not ring homomorphic images of commutative $\mathrm{C}^{*}$-algebras.

Corollary 4. $L^{1}\left(\boldsymbol{R}^{N}\right), A(\boldsymbol{D})$ and $C^{n}([a, b])$ are neither ring homomorphic images of $\ell^{1}(S)$ nor $L^{p}(G)(1 \leq p<\infty$, G a compact abelian group $)$.

Proof. Let $A$ be one of the Banach algebras $\ell^{1}(S)$ and $L^{p}(G)$, and let $B$ be one of the Banach algebras $C^{n}([a, b]), L^{1}\left(\boldsymbol{R}^{N}\right)$ and $A(\boldsymbol{D})$. Then $A$ is a commutative regular Banach algebra which satisfies the condition (\#). Also the Gelfand space of $B$ is a connected infinite set. Assume that there exists a ring homomorphism of $A$ onto $B$, say $\rho$. Then by Theorem $1, \rho$ is either linear or anti-linear and hence continuous. This implies that $\Phi_{A / \operatorname{Ker}(\rho)}$ is homeomorphic to $\Phi_{B}$. But since $\Phi_{A}$ is a discrete space, it follows that $\Phi_{A / \operatorname{Ker}(\rho)}$ is also a discrete space. This is a contradiction.

Q.E.D.

\section{REFERENCES}

[1] B. E. Johnson, The uniqueness of the (complete) norm topology, Bull. Amer. Math. Soc. 73 (1967), 537-539. MR 35:2142

[2] M. Kuczma, An introduction to the theory of functional equations and inequalities, Panstwowe Wydawnictwo Naukowe, Warszawa, 1985. MR 86i:39008

[3] R. Larsen, An introduction to the theory of multipliers, Springer-Verlag, Berlin, 1971. MR 55:8695

[4] R. Larsen, Banach algebras, Marcel Dekker, Inc., New York, 1973. MR 58:7010

[5] L. Molnar, The range of a ring homomorphism from a commutative $\mathrm{C}^{*}$-algebra, Proc. Amer. Math. Soc. 124 (1996), 1789-1794. MR 96h:46090

[6] S.-E. Takahasi and O. Hatori, Commutative Banach algebras and BSE-inequalities, Math. Japonica 37 (1992), 607-614. MR 93h:46069

Department of Basic Technology, Applied Mathematics and Physics, Yamagata UniVERSITY, YONEZAWA 992-8510, JAPAN

Department of Mathematical Science, Graduate School of Science and Technology, Nitgata University, Niigata 950-2102, Japan

E-mail address: hatori@math.sc.niigata-u.ac.jp 